\title{
Sarcoidosis Manifesting as Cardiac Sarcoidosis and Massive Splenomegaly
}

\author{
Sayaka Kawano ${ }^{1}$, Johji Kato ${ }^{2}$, Noriaki Kawano ${ }^{3}$, Yuki Yoshimura ${ }^{4}$, Hiroyuki Masuyama ${ }^{4}$, \\ Takashi Fukunaga ${ }^{4}$, Yoshiya Shimao ${ }^{5}$, Kenroh Mihara ${ }^{6}$, Akira Ueda $^{3}$, Kiyokazu Toyoda ${ }^{7}$, \\ Takuroh Imamura ${ }^{1}$ and Kazuo Kitamura ${ }^{1}$
}

\begin{abstract}
Sarcoidosis is a multisystemic granulomatous disease of unknown etiology. We report an unusual case of sarcoidosis in a woman presenting with cardiac sarcoidosis and massive splenomegaly with a familial history of cardiac sarcoidosis. Cardiac sarcoidosis was diagnosed based on electrocardiogram, echocardiogram, ${ }^{18} \mathrm{~F}-$ fluoro-2-deoxyglucose positron emission tomography $\left({ }^{18} \mathrm{~F}-\mathrm{FDG}-\mathrm{PET}\right)$ and skin histological findings. We performed splenectomy to rule out malignant lymphoma, and histological findings confirmed sarcoidosis. After splenectomy, we initiated prednisolone therapy. After 20 months of diagnosis, she was symptom free. Echocardiography and ${ }^{18}$ F-FDG-PET may be a key diagnostic tool and prednisolone therapy may be safe, effective, and feasible for cardiac sarcoidosis.
\end{abstract}

Key words: cardiac sarcoidosis, massive splenomegaly, pacemaker, splenectomy, prednisolone

(Intern Med 51: 65-69, 2012)

(DOI: 10.2169/internalmedicine.51.5247)

\section{Introduction}

Sarcoidosis is clinically characterized by pulmonary granulomatous lesions. Extrapulmonary granulomatous lesions involve the eye, central nervous system, liver, lymph nodes, spleen, heart, and joints. Massive splenomegaly is quite rare, and in such cases, the presence of lymphoma should be excluded. The incidence of cardiac sarcoidosis ranges from $20 \%$ to $27 \%$ in the United States (1). In Japan, however, the incidence of cardiac sarcoidosis is reported as $58 \%$ (1). Moreover, the prognosis of sarcoidosis with cardiac manifestations has a mortality rate exceeding $40 \%$ at 5 years (2). Here, we report a rare case of a woman who presented with cardiac sarcoidosis and massive splenomegaly with a familial history of cardiac sarcoidosis.

\section{Case Report}

A 58-year-old woman presenting with weight loss, slight anemia, and massive splenomegaly was referred to our hospital in April 2007. Her weight had decreased by $3 \mathrm{~kg}$ during four months. During history-taking, we found that her sister was diagnosed with cardiac sarcoidosis, which was kept under control by a permanent pacemaker. On admission, the patient was normotensive $(120 / 72 \mathrm{mmHg})$ with a heart rate of 72 beats/min. Physical examination revealed erythema nodosum, splenomegaly (4 finger breadths), and no lymphadenopathy. Laboratory findings showed a hemoglobin concentration of $10.6 \mathrm{~g} / \mathrm{dL}$; platelet count of $171 \times$ $10^{9} / \mathrm{L}$; white blood cell count of $3.74 \times 10^{9} / \mathrm{L}$, with $51 \%$ neutrophils, $46 \%$ lymphocytes, and 3\% eosinophils; and serum lactate dehydrogenase (LDH) level of 183 IU/L. Serological tests for hepatitis B virus (HBV), hepatitis C virus (HCV),

\footnotetext{
${ }^{1}$ Department of Internal Medicine, Circulatory and Body Fluid Regulation, Faculty of Medicine, University of Miyazaki, Japan, ${ }^{2}$ Frontier Science Research Center, University of Miyazaki, Japan, ${ }^{3}$ Department of Internal medicine, Miyazaki Prefectural Hospital, Japan, ${ }^{4}$ Department of Cardiology, Miyazaki Prefectural Hospital, Japan, ${ }^{5}$ Department of Pathology, Miyazaki Prefectural Hospital, Japan, ${ }^{6}$ Clinical Laboratory, Miyazaki Prefectural Hospital, Japan and ${ }^{7}$ Department of Surgery, Miyazaki Prefectural Hospital, Japan

Received for publication January 31, 2011; Accepted for publication September 8, 2011

Correspondence to Dr. Sayaka Kawano, sayakawano23@yahoo.co.jp
} 


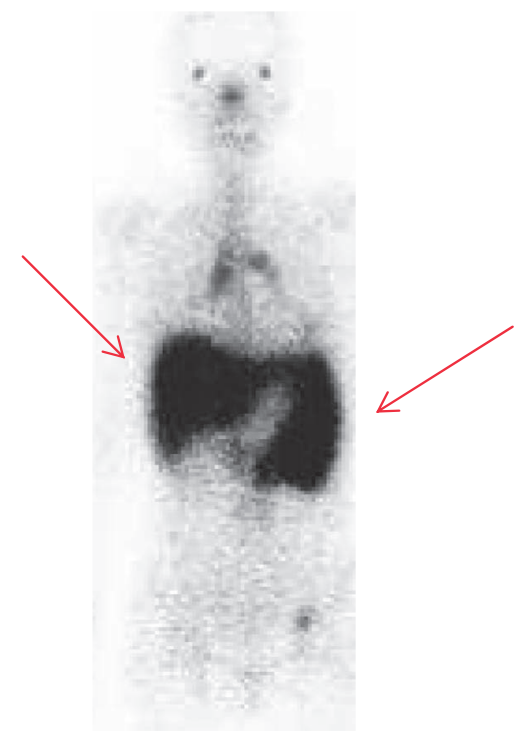

Figure 1. The Ga scintigraphy image showing the uptake by the liver and the spleen.

human T-cell lymphotropic virus type-I (HTLV-I) and antinuclear antibody (ANA) were negative. The serum levels of C3, C4, and CH50 were normal. The serum level of soluble interleukin-2 receptor (sIL-2R), which is considered to represent lymphoma activity, was elevated to $1640 \mathrm{IU} / \mathrm{mL}$. The abdominal computed tomography (CT) scan showed a massive hepatosplenomegaly. In addition to that, gallium (Ga) scintigraphy imaging showed uptake by the liver and spleen (Fig. 1). However, we encountered a difficulty to differentiate the spleen lesion from ominous neoplasms by CT scan and Ga scintigraphy. Splenectomy was planned to confirm the diagnosis of malignant lymphoma, especially splenic marginal zone lymphoma. However, an electrocardiogram (ECG) obtained before splenectomy showed complete right bundle branch block (CRBBB) and left axis deviation (LAD). Moreover, transthoracic echocardiography (TTE) revealed thinning and wall motion abnormalities at the base of the interventricular septum. Left ventricular systolic function was decreased, as the ejection fraction (EF) was $48 \%$ (Fig. 2a, b). Therefore, these findings suggested cardiac sarcoidosis. We further performed the thallium-( $\mathrm{Tl})$ scintigraphy at rest, which revealed patchy perfusion defect at the base of the interventricular septum that was inconsistent with coronary artery distribution (Fig. 3a). The chest CT scan showed mild bilateral lymphadenopathy and no lesion in the lung fields. The serum level of angiotensin-converting enzyme (ACE), which is considered to represent sarcoidosis activity, was elevated to $37.3 \mathrm{IU} / \mathrm{L}$. Hypercalcemia was not observed. The ophthalmological examination revealed uveitis. The findings of ECG, TTE, and Tl scintigraphy strongly supported cardiac sarcoidosis. Therefore, we performed biopsy examination of the erythema nodosum lesion on the face: epithelioid granuloma with multinucleated giant cells of Langhans and no caseous necrosis were detected. These findings were compatible with the diagnosis of sarcoidosis.
We referred to the guidelines for diagnosing cardiac sarcoidosis (3) and diagnosed cardiac sarcoidosis based on ECG findings of CRBBB and LAD, TTE findings of thinning and wall motion abnormalities at the base of the interventricular septum and $\mathrm{Tl}$ scintigraphy findings of the patchy perfusion defect.

To evaluate cardiac sarcoidosis, we performed ${ }^{18} \mathrm{~F}$-fluoro2-deoxyglucose positron emission tomography $\left({ }^{18} \mathrm{~F}-\mathrm{FDG}-\right.$ PET)(Fig. 3b). ${ }^{18}$ F-FDG-PET revealed the focal on diffuse uptake pattern of the heart, while Ga scintigraphy did not show abnormal cardiac uptake despite planner analysis in our case. A cardiac biopsy was not performed in this case due to the presence of an abnormal PET finding. On the basis of all these findings, we diagnosed sarcoidosis as the disease responsible for the skin lesion, ocular lesion, and cardiac lesion. However, the spleen was also considered to be a possible site of lymphoma and sarcoidosis. To exclude this possibility, we performed splenectomy following transplantation of a transient pacemaker in July 2007. The spleen was huge $(21 \mathrm{~cm} \times 15 \mathrm{~cm} \times 10 \mathrm{~cm})$ (Fig. $4 \mathrm{a}, \mathrm{b})$. The histological findings revealed epithelioid granuloma with multinucleated giant cells of Langhans, asteroid body, and no caseous necrosis (Fig. 4c, d). These findings confirmed sarcoidosis.

According to the guidelines of sarcoidosis in Japan (3), the present case was that of histologically proven sarcoidosis of the skin and spleen. Finally, we confirmed that the sarcoidosis involved the eye, heart, abdominal lymph nodes, skin, spleen, and liver. We further examined the human leukocyte antigen (HLA)-type and our patient also showed the presence of HLA-DQB $1{ }^{*} 060$.

According to the guidelines for the treatment of cardiac sarcoidosis (4), we administered $30 \mathrm{mg} /$ day prednisolone (PSL) to improve the cardiac and ocular lesion. After splenectomy and PSL therapy, the ACE and sIL-2R levels decreased. Prednisolone was gradually tapered because left ventricular systolic function was improved $(\mathrm{EF} 48 \% \rightarrow 55 \%)$. However, the finding of ECG did not show remarkable change between before and after prednisolone treatment. Candida oralitis and Diabetes mellitus (DM) were complications that developed due to the PSL therapy; these complications were tolerable and controlled by the administration of fluconazole and insulin therapy, respectively. At this time, 20 months after the diagnosis of sarcoidosis, she had no symptoms and cardiac sarcoidosis was under control with a maintenance dose of $5 \mathrm{mg}$ PSL.

\section{Discussion}

Sarcoidosis is a multisystemic granulomatous disease of unknown etiology. Granulomatous lesions of the spleen are common in sarcoidosis; however, massive splenomegaly is quite rare $(5,6)$. Mohan et al summarized the clinical features of 17 cases of massive splenomegaly from sarcoidosis (5). Fordice et al reported 628 patients with quantified splenomegaly and that only 20 of 6,074 cases of sarcoidosis (3\%) had massive splenomegaly (6). To the best of our 

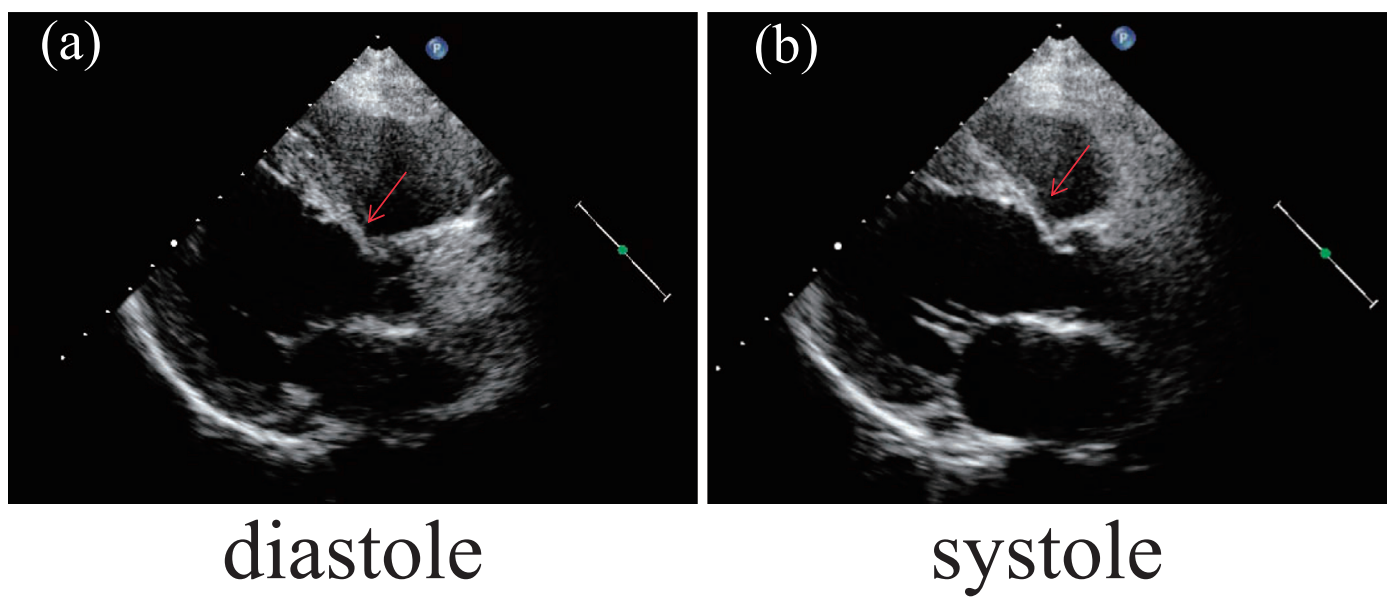

Figure 2. a, b. Transthoracic echocardiography image showing the thinning at the base of the interventricular septum.

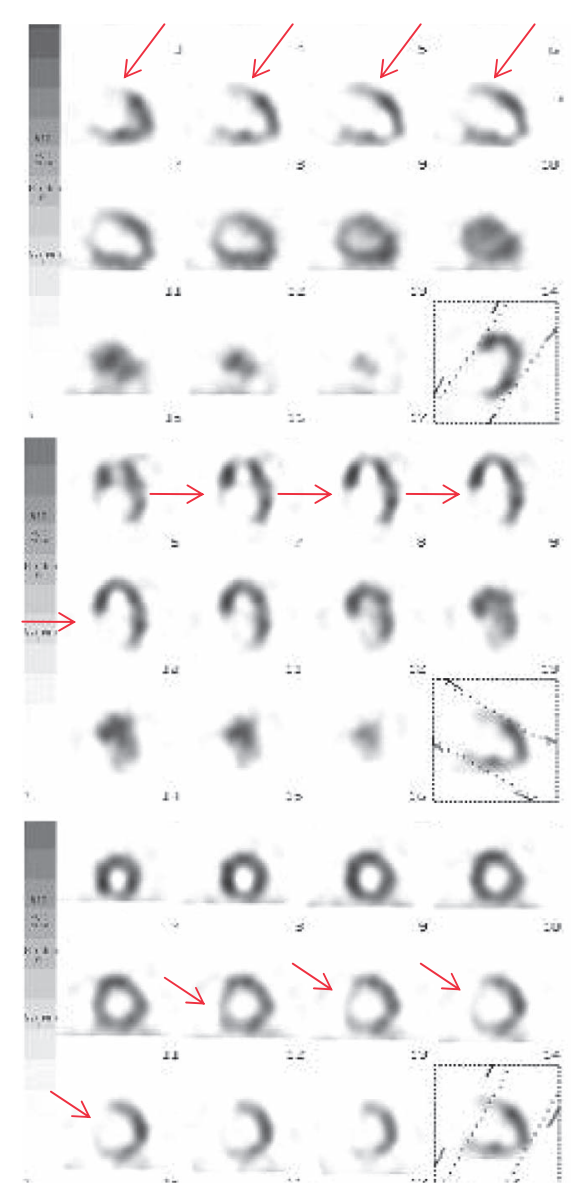

(a)

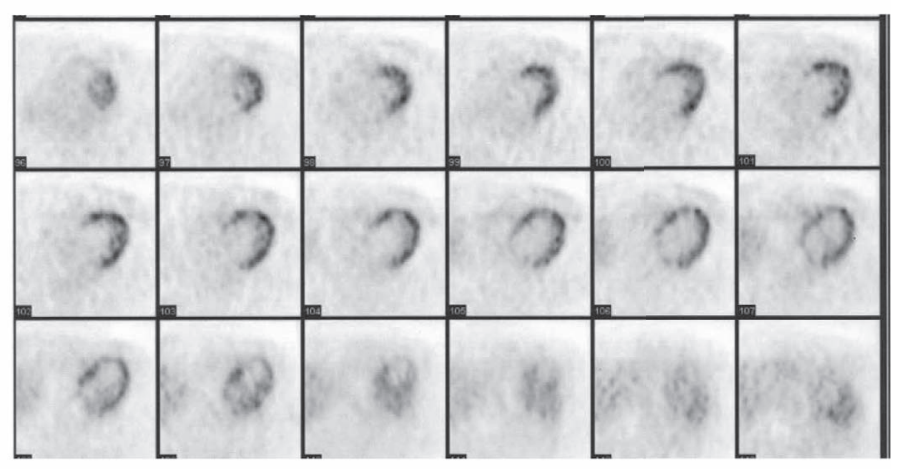

(b)

Figure 3. a. The thallium-201 scan at rest showing the patchy perfusion defect at the base of the interventricular septum. b. ${ }^{18}$ F-FDG-PET image revealed the focal on diffuse uptake pattern of the heart.

knowledge, this is the first case of sarcoidosis in a woman who presented with massive splenomegaly and cardiac sarcoidosis with a familial history of cardiac sarcoidosis.

First, the key to the diagnosis of sarcoidosis is the ECG findings of CRBBB and LAD and TTE findings of thinning and wall motion abnormalities at the base of the interventricular septum. Recently, Okumura et al reported that abnormal ${ }^{18} \mathrm{~F}$-FDG uptake on fasting ${ }^{18} \mathrm{~F}$-FDG PET is seen in active inflammatory lesions at the early stage of cardiac sarcoidosis (7). Moreover, Ishimaru et al reported that focal 

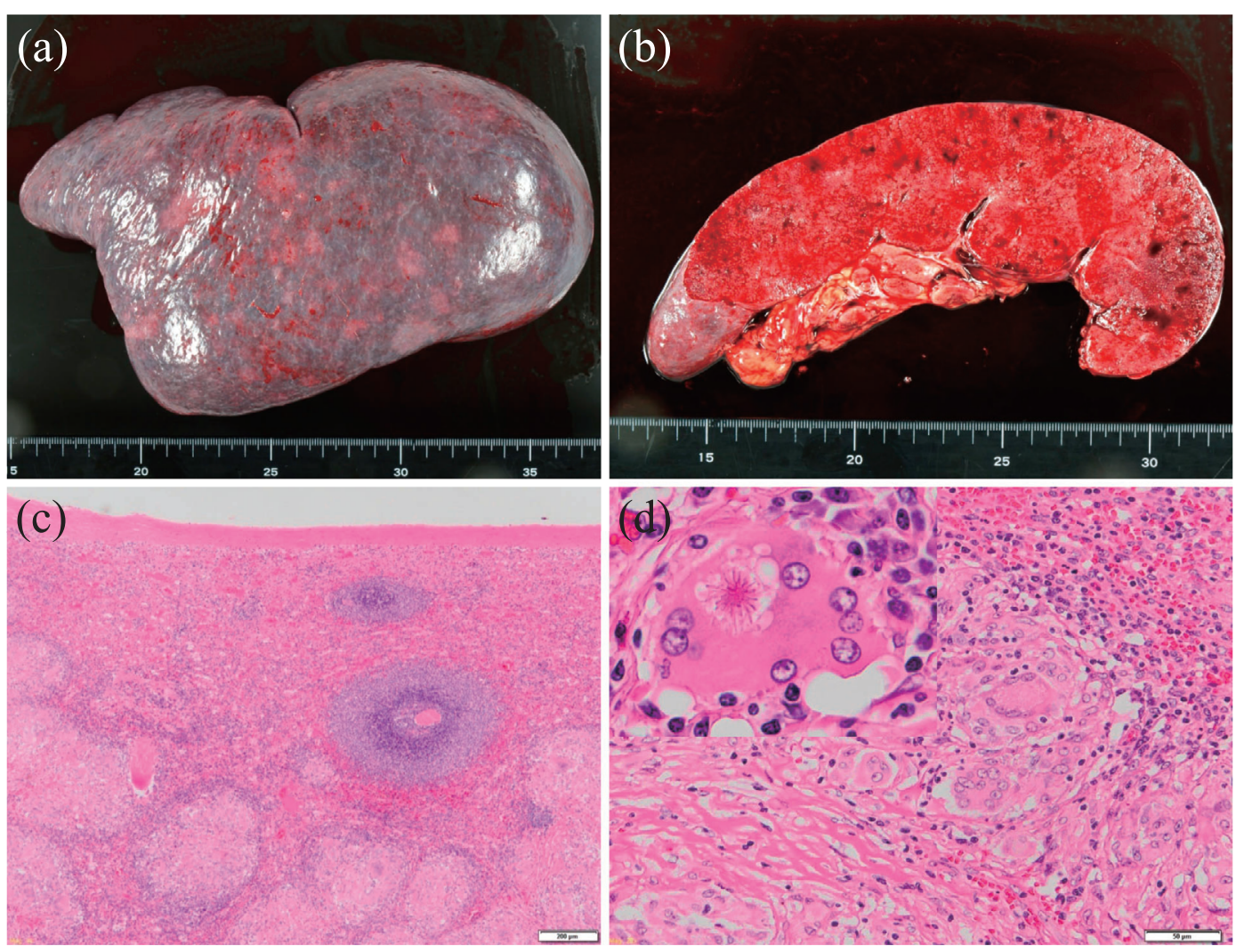

Figure 4. a, b. The spleen was huge and was $21 \mathrm{~cm} \times 15 \mathrm{~cm} \times 10 \mathrm{~cm}$. c, d. Histological findings of the spleen specimen. Abundant compact epithelioid granulomas with multinucleated giant cells of Langhans type were seen. Some of these giant cells had asteroid bodies. No caseous necrosis was seen in these granulomas. These granulomas were also present in the lymph nodes.

and focal on diffuse uptake pattern of the heart on ${ }^{18} \mathrm{~F}$-FDGPET images is a characteristic feature of cardiac sarcoidosis patients (8). In the present case, consistent with previous reports $(7,8)$, the characteristic pattern of abnormal finding of ${ }^{18}$ F-FDG PET image was also useful to make the diagnosis.

Naruse et al reported that susceptibility of cardiac sarcoidosis in Japanese females may be related to the presence of HLA-DQB1 060 (9). Therefore, we examined the human leukocyte antigen (HLA)-type to clarify the relationship between HLA and the occurrence of cardiac sarcoidosis. The present patient also showed the presence of HLADQB1 ${ }^{*} 060$. Moreover, the occurrence of cardiac sarcoidosis in the same family, as in our patient and her sister, indicated the possibilities of accumulation in the progression of cardiac sarcoidosis.

Although we examined the etiology of splenomegaly by various imaging techniques, it was difficult to determine lymphoma and sarcoidosis. Massive splenomegaly from sarcoidosis can mimic more ominous neoplasms, such as lymphoma and/or metastasis. The so-called sarcoidosislymphoma syndrome develops as a consequence of immunological abnormalities observed in sarcoidosis $(5,6)$, and splenectomy was performed because of lymphoma/metastasis and rupture. In the present patient, we performed splenectomy in order to exclude lymphoma/metastasis. The histological examination of the resected specimen revealed splenic sarcoidosis. Splenectomy may be helpful to diagnose massive splenomegaly from sarcoidosis and to determine therapeutic ground strategy. Moreover, Sharma et al, in their long follow-up study reported that 17 patients of splenic sarcoidosis who underwent splenectomy had no severe infectious complications; 13 patients with splenectomy were followed up for a period of 2 to 36 years and there was no evidence of post-splenectomy sepsis, pneumonia, or disseminated infection (10). The present patient also suffered from Candida oralitis and was therefore administered fluconazole.

Furthermore, corticosteroid therapy was initiated against cardiac and ocular sarcoidosis. Owing to the lack of randomized trials, corticosteroids are used as the first-line therapeutic agents in cardiac sarcoidosis on the basis of the relatively large published clinical reports (1). Yazaki et al reported that cardiac sarcoidosis patients with PSL therapy have a better prognosis than those without therapy (2). There is also no evidence on the appropriate initiating dose of corticosteroids. The prognosis of cardiac sarcoidosis with PSL therapy exhibits no difference between doses of $30 \mathrm{mg} /$ day and over $40 \mathrm{mg} / \mathrm{day}$, and an initial dose of $30 \mathrm{mg} / \mathrm{day}$ or $60 \mathrm{mg}$ every other day has proven effective (2). This dose can be tapered over a period of 6-12 months. A maintenance dose of $5-10 \mathrm{mg} / \mathrm{day}$ for a prolonged period is necessary to prevent relapse of cardiac sarcoidosis.

In conclusion, here we described a rare case of splenic 
and cardiac sarcoidosis with a familial history of cardiac sarcoidosis. This case indicated that splenectomy is beneficial in the definite diagnosis of splenic sarcoidosis, and immunosuppressive treatment with PSL may stabilize and prevent the progression of cardiac sarcoidosis and also reduce the risk of sudden death. Echocardiograms are extremely useful to attain the precise diagnosis of cardiac sarcoidosis. Future studies on the type of immunosuppressive agents suitable for cardiac sarcoidosis is necessary.

The authors state that they have no Conflict of Interest (COI).

\section{References}

1. Bargout R, Kelly RF. Sarcoid heart disease: clinical course and treatment. Int J Cardiol 97: 173-182, 2004.

2. Yazaki Y, Isobe M, Hiroe M, et al; Central Japan Heart Study Group. Prognostic determinants of long-term survival in Japanese patients with cardiac sarcoidosis treated with prednisone. Am J Cardiol 88: 1006-1010, 2001.

3. Sugisaki K. Diagnostic guidelines and criteria for sarcoidosis-
2006. Nihon Kokyuki Gakkai Zasshi 46: 768-780, 2008 (in Japanese).

4. Hiramitsu S, Morimoto S, Uemura A, et al. National survey on status of steroid therapy for cardiac sarcoidosis in Japan. Sarcoidosis Vasc Diffuse Lung Dis 22: 210-213, 2005.

5. Mohan A, Sood R, Shariff N, Gulati MS, Gupta SD, Dutta AK. Sarcoidosis manifesting as massive splenomegaly: a rare occurrence. Am J Med Sci 328: 170-172, 2004.

6. Fordice J, Katras T, Jackson RE, et al. Massive splenomegaly in sarcoidosis. South Med J 85: 775-778, 1992.

7. Okumura $\mathrm{W}$, Iwasaki $\mathrm{T}$, Toyama $\mathrm{T}$, et al. Usefulness of fasting ${ }^{18} \mathrm{~F}$ FDG PET in identification of cardiac sarcoidosis. J Nucl Med 45: 1989-1998, 2004.

8. Ishimaru S, Tsujino I, Takei T, et al. Focal uptake on ${ }^{18} \mathrm{~F}$-fluoro-2deoxyglucose positron emission tomography images indicates cardiac involvement of sarcoidosis. Eur Heart J 26: 1538-1543, 2005.

9. Naruse TK, Matsuzawa Y, Ota M, et al. HLA-DQB1 ${ }^{*} 0601$ is primarily associated with the susceptibility to cardiac sarcoidosis. Tissue Antigens 56: 52-57, 2000.

10. Shama OP, Vucinic V, James DG. Splenomegaly in sarcoidosis: indications, complications, and long-term follow-up. Sarcoidosis Vasc Diffuse Dis 19: 66-70, 2002.

(C) 2012 The Japanese Society of Internal Medicine http://www.naika.or.jp/imindex.html 